Slavica

bruxellensia

\section{Slavica bruxellensia}

Revue polyphonique de littérature, culture et histoire

slaves

$5 \mid 2010$

Après 1989

\title{
Sylvain Savoia et Małgorzata Sowa, Marzi : 1989...
}

\section{Dariusz Vanhonnaeker}

\section{OpenEdition \\ Journals}

Édition électronique

URL : http://journals.openedition.org/slavica/316

DOI : $10.4000 /$ slavica.316

ISSN : 2034-6395

Éditeur

Université libre de Bruxelles - ULB

Édition imprimée

Pagination : 88-89

ISSN : 2031-7654

Référence électronique

Dariusz Vanhonnaeker, « Sylvain Savoia et Małgorzata Sowa, Marzi : 1989... », Slavica bruxellensia [En ligne], 5 | 2010, mis en ligne le 09 juillet 2012, consulté le 22 septembre 2020. URL : http:// journals.openedition.org/slavica/316 ; DOI : https://doi.org/10.4000/slavica.316

Ce document a été généré automatiquement le 22 septembre 2020.

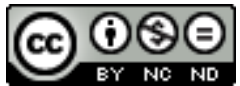

Les contenus de Slavica bruxellensia sont mis à disposition selon les termes de la Licence Creative Commons Attribution - Pas d'Utilisation Commerciale - Pas de Modification 3.0 France. 


\section{Sylvain Savoia et Małgorzata Sowa, Marzi : 1989...}

\section{Dariusz Vanhonnaeker}

\section{RÉFÉRENCE}

Sylvain Savoia et Małgorzata Sowa, Marzi : 1989..., Marcinelle, Dupuis, 2009, 238 p. 
1 «Je suis Marzi, je raconte Marzi, mais Marzi n'est pas que mon histoire. Néanmoins, je n'incarne pas la Pologne, ni l'histoire de la Pologne, je raconte juste ma version, mes souvenirs, tout est subjectif, tout est mien, je ne prétends rien, j'essaie de rester moi-même et raconter le monde à travers moi-même, le bleu-gris de mes yeux, mes lentilles. »

2 Fruit de la collaboration d'une scénariste polonaise et d'un dessinateur français, ce deuxième volume de l'édition intégrale des cinq albums, retrace l'enfance de Marzi du printemps 1988 à l'été 1989. Jonglant avec les épisodes historiques et la vie quotidienne de l'héroïne éponyme, les

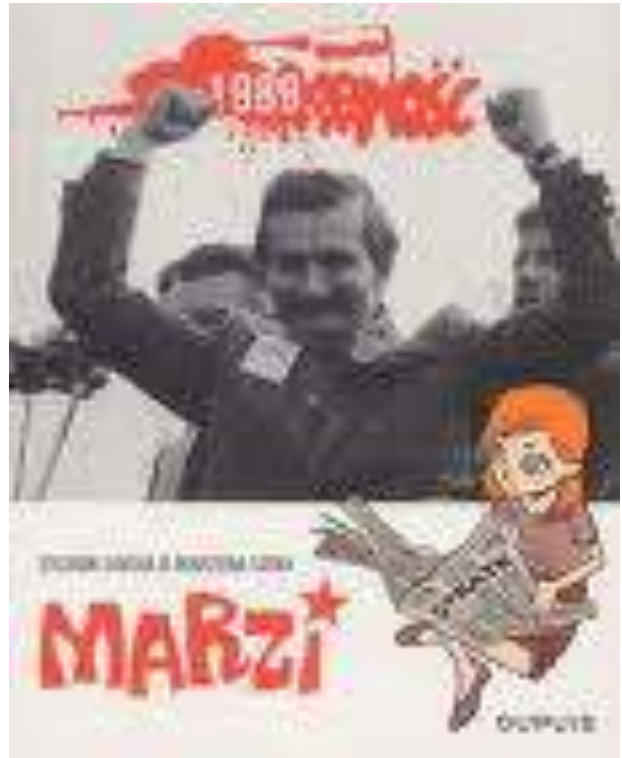
dix-neuf chapitres de ce second opus nous font découvrir ou redécouvrir les changements profonds auxquels est alors en proie la société polonaise.

3 Revisitant un passé dont on fête aujourd'hui les vingt ans, Marzi nous donne une vision originale de ce qu'était à l'époque, la vie d'une petite fille de l'autre côté du Mur. Entre l'éclosion de Solidarność et ses excursions à la campagne, entre les files d'attentes et la peur de ne pas voir revenir son père en grève, un monde complexe, parfois obscur se révèle à elle. Marzi ne comprend pas tout ce qui arrive. Mais elle le ressent, et décline de la sorte les incertitudes véhiculées par cette première faille fissurant le bloc communiste. Toutefois, il ne s'agit pas d'un récit historique. Que ce soit la Table Ronde ou la chute du Mur, l'Histoire est avant tout vécue à travers l'histoire de Marzi, de ce qu'elle devine, perçoit ou redoute, de ses impressions d'enfant grandissant en même temps que renaît, petit à petit, la Pologne. Ainsi, outre sa fonction testimoniale, le récit invite à repenser un monde où les traumatismes d'hier ouvrent sur demain, et où l'espoir de voir s'accomplir les rêves d'une enfant répond à la morosité du quotidien.

fatigue et la colère s'incrustent partout. (...) Et nous, les enfants, nous absorbons tout. Nous nous laissons imbiber de tout sans que les gens s'en aperçoivent... Jusqu'au moment où on nous presse. Nous sommes des enfants éponges. Il ne suffit pas d'essorer, il faut faire attention dans quoi on nous plonge. Même lavés, rincés, séchés à maintes reprises, les traces restent en nous. "

Ce «nous» réapparaissant tout au long du récit, ouvre sur une dimension polyphonique particulièrement intéressante dans Marzi, et élargit par ailleurs considérablement le champ d'expression habituellement attribué à la bande dessinée. Partagé entre deux langues (le français et le polonais), entre deux âges (l'enfance et le recul de l'âge adulte) ou encore entre deux entités personnelles (le "moi » et un « nous » polyvalent, renvoyant tantôt à sa génération, tantôt au peuple polonais ou à la population ouvrière de Stalowa Wola), le texte joue sur différents niveaux de narration. Cependant, cette polyphonie énonciative n'engendre pas de réelle diffraction. Au contraire, l'instance narratrice fédère, par la transversalité de son regard, une situation historique à la fois personnelle et collective inscrivant son récit dans une dynamique plus vaste de reconstruction mémorielle et d'appropriation de l'Histoire. À la 
découverte d'une page du $\mathrm{XX}^{\mathrm{e}}$ siècle, ce livre s'adresse donc à tous, enfants ou adultes curieux d'entrevoir ce qu'était la vie derrière le rideau de fer. Guidés par la fraîcheur d'un point de vue contrastant avec la morosité des années Jaruzelski, les auteurs conjuguent la qualité graphique à celle du texte. Ils nous emmènent dans un passé oscillant entre le rouge et le gris, sans jamais tomber dans le diabolisme ni les pièges du misérabilisme. Perspicace et spontané, le regard de Marzi séduit et emporte le lecteur dans un tourbillon d'évènements, plus ou moins importants mais jamais ordinaires.

INDEX

Index géographique : Pologne

Index chronologique : communisme, XXe siècle

Mots-clés : bande-dessinée

\section{AUTEURS}

\section{DARIUSZ VANHONNAEKER}

Étudiant à l'Université Libre de Bruxelles en Langues et Littératures françaises et romanes 